\title{
Sex-differential DNA methylation and associated regulation networks in human brain implicated in the sex-biased risks of psychiatric disorders
}

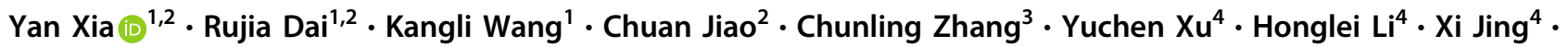 \\ Yu Chen ${ }^{1} \cdot$ Yi Jiang ${ }^{1,5} \cdot$ Richard F. Kopp ${ }^{2} \cdot$ Gina Giase $^{6} \cdot$ Chao Chen $^{1,2,7} \cdot$ Chunyu Liu $\mathbb{D}^{1,2,8}$
}

Received: 8 November 2018 / Revised: 18 March 2019 / Accepted: 22 March 2019 / Published online: 11 April 2019

(C) Springer Nature Limited 2019

\begin{abstract}
Many psychiatric disorders are characterized by a strong sex difference, but the mechanisms behind sex-bias are not fully understood. DNA methylation plays important roles in regulating gene expression, ultimately impacting sexually different characteristics of the human brain. Most previous literature focused on DNA methylation alone without considering the regulatory network and its contribution to sex-bias of psychiatric disorders. Since DNA methylation acts in a complex regulatory network to connect genetic and environmental factors with high-order brain functions, we investigated the regulatory networks associated with different DNA methylation and assessed their contribution to the risks of psychiatric disorders. We compiled data from 1408 postmortem brain samples in 3 collections to identify sex-differentially methylated positions (DMPs) and regions (DMRs). We identified and replicated thousands of DMPs and DMRs. The DMR genes were enriched in neuronal related pathways. We extended the regulatory networks related to sex-differential methylation and psychiatric disorders by integrating methylation quantitative trait loci (meQTLs), gene expression, and protein-protein interaction data. We observed significant enrichment of sex-associated genes in psychiatric disorder-associated gene sets. We prioritized 2080 genes that were sex-biased and associated with psychiatric disorders, such as NRXN1, NRXN2, NRXN3, FDE4A, and SHANK2. These genes are enriched in synapse-related pathways and signaling pathways, suggesting that sexdifferential genes of these neuronal pathways may cause the sex-bias of psychiatric disorders.
\end{abstract}

This work was honored Hugh Gurling Memorial Awards by the International Society of Psychiatric Genetics at XXIV World Congress of Psychiatric Genetics and Charles J. Epstein Trainee Award for Excellence in Human Genetics Research (Semifinalist) at 67th Annual Meeting of the American Society of Human Genetics.

Supplementary information The online version of this article (https:// doi.org/10.1038/s41380-019-0416-2) contains supplementary material, which is available to authorized users.

Chao Chen

chenchao@sklmg.edu.cn

$\triangle$ Chunyu Liu

liuch@upstate.edu

1 Center for Medical Genetics, School of Life Sciences, Central South University, Changsha, Hunan, China

2 Department of Psychiatry, State University of New York Upstate Medical University, Syracuse, NY, USA

3 Department of Neuroscience, State University of New York Upstate Medical University, Syracuse, NY, USA

\section{Introduction}

Many psychiatric disorders are characterized by a strong sexual difference including different prevalence, age of onset, symptom severity, and responses to medications. For example, males are 3-4 times more likely to develop autism spectrum disorder (ASD) [1, 2], and typically have an earlier age of onset and a worse course of treatment for

4 Xiangya School of Medicine, Central South University, Changsha, Hunan, China

5 Genetics Institute, Vanderbilt University School of Medicine, Nashville, TN, USA

6 Department of Public Health, University of Illinois at Chicago, Chicago, IL, USA

7 National Clinical Research Center for Geriatric Disorders, the Xiangya Hospital, Central South University, Changsha, Hunan, China

8 School of Psychology, Shaanxi Normal University, Xi' an, China 
schizophrenia (SCZ) [3]. Females are 2-3 times more likely to develop major depression disorder (MDD) [4] and exhibit greater symptom severity, greater functional impairment, more atypical depressive symptoms and higher rates of comorbid anxiety [5]. Understanding the basis of sex difference in these disorders can provide important insights into their etiology and offer an opportunity to deliver sex-specific treatments and care.

At least four models have been proposed to explain sex bias of psychiatric diseases [6-8]: specific susceptibility genes that reside on the $\mathrm{X}$ or $\mathrm{Y}$ chromosome [9], differential genetic liability thresholds between the sexes [7], major influences of hormonal levels in the sexes [10], and genesex interactions [11]. A recent study that systematically evaluated the four models proposed that genetic-environmental interaction has a strong contribution of sex bias in psychiatric disorders [12]. However, the molecular mechanisms that link genetic-environmental factors to sexbiased phenotypes are unknown.

Epigenetics is the product of genetic and environmental influences [13], thus epigenetic modifications of DNA are attractive candidates for explaining sexual differences. DNA methylation, the best-studied type of DNA modification, has been reported to play important roles in sexually differential characteristics of the human brain [14-29]. For example, McCarthy et al. [26] conducted a meta-analysis on multiple tissues including brain and found sex-specific methylated genes related to immune response, RNA splicing, and DNA repair. Xu et al. [21] reported sexspecific methylated genes that participate in ribosome structure and function, RNA binding, and protein translation in adult postmortem prefrontal cortex. Spiers et al. [21] analyzed sex-differential methylation in fetal brain and found a highly significant correlation with results from $\mathrm{Xu}$ et al., indicating that most sex differences in the brain methylome occur early in fetal development and are stable throughout life. However, these studies only focused on DNA methylation and did not study the regulatory networks associated with this epigenetic modification. It is unknown whether or how these regulatory components, which contain upstream genetic regulators [30] and a cascade of downstream gene expression and associated protein networks, could influence sex bias of psychiatric disorders.

The purpose of this study is to describe the landscape of sex-differential DNA methylation, explore its regulatory networks, and evaluate their potential involvement in psychiatric disorders. Our hypotheses are: (1) sex-differences exist at both DNA methylation and its regulatory networks; (2) psychiatric disorder-related genes have different methylation levels or different methylation regulation between male and female. We compiled data from 1408 postmortem brain samples from three collections and investigated sex-associated individual $\mathrm{CpG}$ loci (differentially methylated positions, DMPs) and genomic regions (differentially methylated regions, DMRs). Then we investigated the related genetic, transcriptomic and proteomic regulatory networks of DMPs or DMRs. Further, we explored their contribution to the sex bias of psychiatric disorders. We found 2080 genes with sex-differential methylation that have been previously associated with psychiatric disorders. These genes are enriched in synapserelated and signaling pathways.

\section{Materials and methods}

To systematically explore sex-differential DNA methylation profiles and related regulatory networks in human brain, we obtained data of 1408 human postmortem brain samples from three collections, the Religious Orders Study and the Rush Memory and Aging Project (ROSMAP) [30], Jaffe et al. [19], and Horvath et al. [31]. (Fig. 1). The ROSMAP dataset was generated from dorsolateral prefrontal cortex (DLPFC) of 698 nonpsychiatric controls which contained 227 males and 471 females. For the collection of Jaffe et al., we used the DLPFC data from 450 controls without any known history of psychiatric disorders (158 female, 292 males) across the lifespan. For the collection of Horvath et al., 260 control samples (130 females, 130 males) from multiple brain regions were collected, including caudate nucleus $(n=$ $12)$, cingulate gyrus $(n=12)$, cerebellum $(n=32)$, frontal cortex $(n=41)$, hippocampus $(n=25)$, midbrain $(n=$ $18)$, motor cortex $(n=12)$, occipital cortex $(n=33)$, parietal lobe $(n=23)$, sensory cortex $(n=12)$, temporal cortex $(n=29)$, and visual cortex $(n=11)$ [31].

\section{DNA methylation data}

DNA methylation was characterized using Illumina HumanMethylation450 BeadChips to interrogate more than 485,000 methylation sites in the three collections. Raw data (idat format) were provided by both ROSMAP and Jaffe et al. (GSE74193), while the $\beta$ value matrix was provided by Horvath et al. (GSE64509). We used the ROSMAP data as the discovery dataset for sex-differential DNA methylation profiling since it had the largest sample size. GSE74193 and GSE64509 were used as the replication datasets. We used data from all the brain regions in GSE64509 to replicate the results from discovery dataset.

\section{Gene-expression data}

Gene-expression data were obtained from ROSMAP samples using RNA-sequencing from DLPFC of 540 individuals (a subset of DNA methylation samples). Gene-expression 
Fig. 1 Overview of the study design

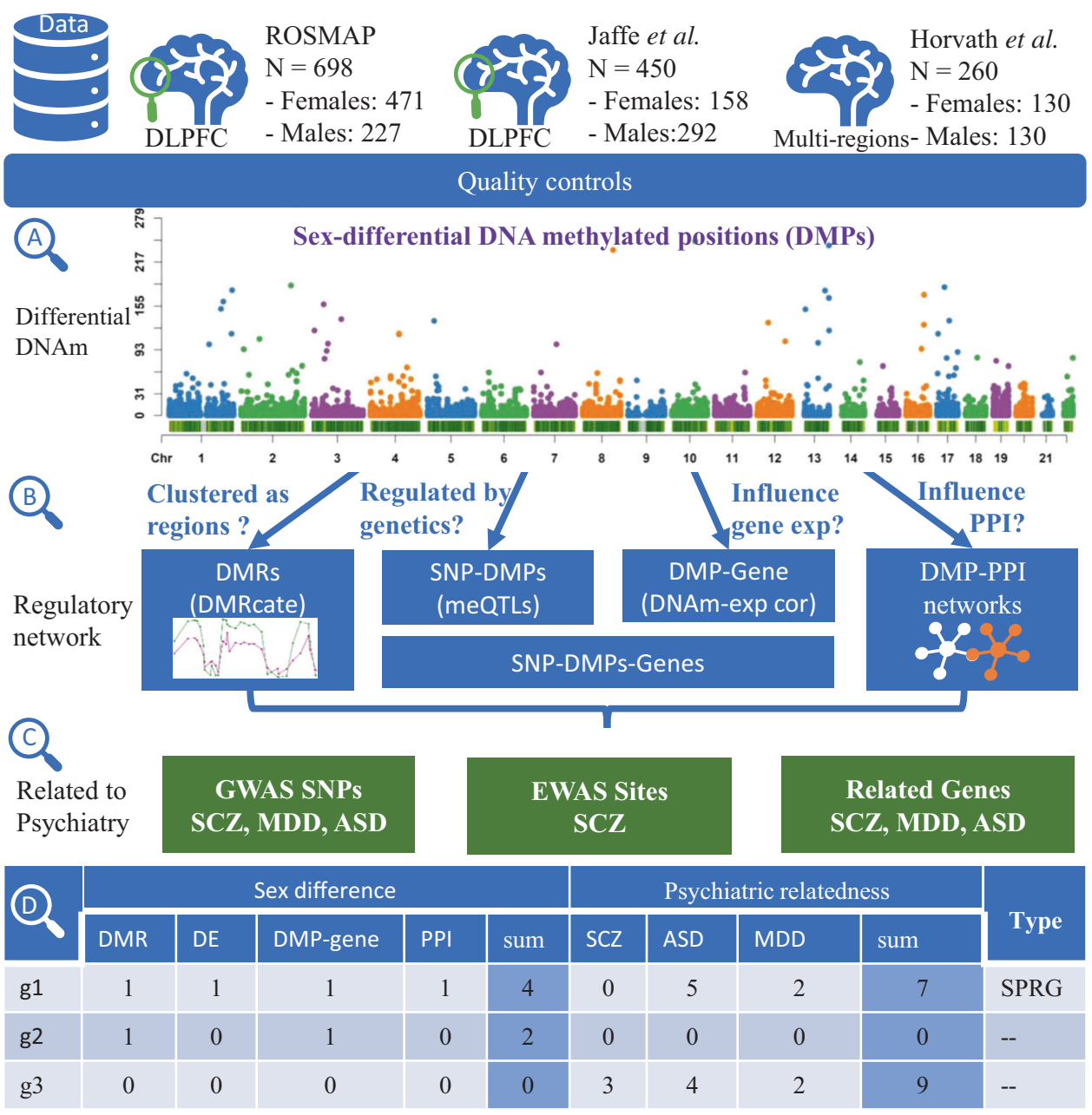

data were normalized using fragments per kilobase of transcript per million (FPKM) values. Detailed descriptions of data acquisition, RNA-seq protocols, and the process pipeline are as described previously [32].

\section{MeQTL data}

The meQTL data were obtained from the Jaffe et al. and ROSMAP. In the ROSMAP study, $\mathrm{Ng}$ et al. performed meQTL mapping between single polynucleotide polymorphisms (SNPs) and methylation in $5 \mathrm{~kb}$ windows among 463 individuals. In total 9,939,236 SNPmethylation pairs were tested which contained 2,358,873 SNPs and 412,152 $\mathrm{CpG}$ sites, resulting in 693,696 significant meQTL pairs $(383,920$ SNPs with $56,973 \mathrm{CpG}$ sites) using a Bonferroni corrected $p$ value threshold (adj. $p<0.05$, two-tailed) (detailed procedure of meQTL mapping is described previously [32]). Jaffe et al. performed meQTL mapping in $20 \mathrm{~kb}$ windows among 258 individuals. In total 7,426,085 SNPs and 477,636 CpG sites were analyzed, resulting in 4,107,214 significant meQTLs at a false-discovery rate $(\mathrm{FDR})<1 \%$. In this study, we combined these two meQTL datasets, and used only the reproducible meQTLs pairs that were statistically significant in both datasets.

\section{Protein-protein interaction data}

The protein-protein interaction (PPI) data, for building downstream regulatory network, was derived from the Pathway Commons resource [33] based on the procedure described by West et al. [34]. The PPI network consists of 8434 genes (annotated to NCBI Entrez identifiers) and 303,600 interactions.

\section{Quality control and preprocessing}

We used the $\mathrm{R}$ package ChAMP (version 1.2.1) [35] to process the raw idat format methylation data. The function champ.load was used to remove probes meeting the following criteria: (1) probes with a detection $p$ value above 0.01 in one or more samples; (2) probes with beads count $<3$ in at least $5 \%$ of samples; (3) probes with SNPs as identified in Zhou et al. [36]; and (4) probes that align to 
multiple locations as identified in Zhou et al. [36]. Probes with a $\beta$ value of 0 were replaced with $1.00 \mathrm{e}-6$, and probes with missing $\beta$ values were imputed using a k-nearest neighbor algorithm by impute.knn function in impute package [37]. Samples with more than $1 \%$ of probes filtered were removed. We next used beta mixture quantile dilation (BMIQ) in function champ.norm to adjust the $\beta$ values of type II probes into a statistical distribution characteristic of type I probes, which has previously been shown to best minimize the variability between replicates [38]. After BMIQ normalization, we further filtered the probes based on the high-quality probes [39] defined by Naeem et al. Probes were removed when they had: (1) the variants based on the 1000 Genomes database, (2) small insertions and deletions, (3) repetitive DNA, and (4) regions with reduced genomic complexity that may affect probe hybridization.

Considering the impact of variable cell-types' compositions on DNA methylation, we calculated the cell-type compositions of the brain tissue using a reference-based method, RefbaseEWAS [40]. We downloaded DNA methylation reference data from 28 control brains, that had been processed by fluorescence activated cell sorting to extract different cell types [41]. We calculated cell-type proportions and used the values as covariates in further analysis. We applied the singular value decomposition method (SVD) [42] to identify unknown covariates. ComBat function was used to correct batch effects and position effects [43-45]. Other confounders such as age and postmortem interval were controlled using a linear regression model. Confounder removal was confirmed by surrogate variable analysis [44].

Quality control for gene-expression data involved selecting genes with FPKM $>0.1$ in at least ten samples which removed the low-expressed genes. Potential confounders such as batch effects, age, and cell component were removed by SVD. We used the $\log 2$ transformed FPKM value for further association analysis.

\section{Sex-differentially methylated positions and regions}

After removal of all confounders, statistical analysis was implemented to identify sex-DMPs and regions (DMRs). Since the $M$-values ( $\log 2$ ratio of the intensities of methylated probe versus unmethylated probe) are more statistically valid for the differential analysis of methylation levels [46], we calculated the $M$ value from the $\beta$ value and used the $M$ value to calculate the differential methylation signal between males and females using limma [47]. After correcting the multiple test burden, we defined the features with FDR $<0.05$ as DMPs (Fig. 1a). To detect the sexdifferential DNA methylation regions, we used the DMRfinding algorithm DMRcate [48], which clustered the groups of significant probes $($ FDR $<0.05)$ within $1 \mathrm{~kb}$ as
DMR, and excluded DMRs containing less than three CpG sites.

\section{Sex-differential regulatory network}

To comprehensively understand the DNA methylation regulatory network, we integrated upstream genetic regulators, downstream gene expression, and protein-protein intereraction (PPI) networks with DMPs and DMRs. The meQTL data from both ROSMAP and GSE74193, gene expression from ROSMAP, and PPI data from Pathway Common were used in this regulatory network (Fig. 1b). We searched for DMPs and potential upstream regulators using reproducible meQTLs from ROSMAP and GSE74193 formed as SNP-DMP pairs. Then we tested the association of DMPs with gene expression by calculating the Spearman correlation between the methylation level of DMPs ( $\beta$ value) and the expression levels of nearby genes $(10 \mathrm{~kb})$. This calculation was based on methylation and expression data from 468 brain samples (ROSMAP methylation and expression profiling). FDR was used for multiple testing correction. The associated DMP-gene pairs were defined using absolute value of correlation coefficient $>0.3$ and FDR $<0.05$. Then, using the DMPs as index, we connected the SNP-DMP and the DMP-gene pairs to SNPDMP-Gene groups.

\section{Protein-protein interaction subnetwork related to sex-differential DNA methylation}

We used a functional supervised algorithm, functional epigenetic modules (FEM) [49] to identify subnetworks containing genes exhibiting sex-related differential DNA methylation in promoter regions. Using probe-level analysis by the champ.EpiMod function, the most differentially methylated probe was assigned to each gene, and the PPI subnetworks which inferred the differential methylated module was extracted.

\section{Overrepresentation of psychiatric disorder-related signals in sex-differential loci}

To explore whether genes associated with psychiatric disorder show sex-differential methylation or regulation, we tested for enrichment between sexually different DNA methylation genes and SCZ-, ASD-, and MDD-associated genes/loci. We completed a series of comparisons at the SNPs, CpGs, gene, and protein levels (Fig. 1c).

For SNPs level comparisons, we investigated whether genome-wide significant SNPs associated with SCZ, ASD, and MDD were enriched in SNPs which regulated DMPs (SNP-DMP pairs from meQTLs). For CpG-level comparison, we tested whether $\mathrm{CpG}$ sites that were 
associated with diseases from epigenome-wide association studies (EWAS) were enriched in DMPs. For the gene level comparisons, we determined if genes associated with SCZ, ASD, and MDD show sex-differential manner. The sex-differential genes contained DMR genes, DMP associated expression genes, sex-differential expression genes, and genes in the sex-related PPI network. The disorder-related genes came from genetic association, differential expression and co-expression studies. Due to data availability limitations, we studied SCZ, ASD, and MDD separately in SNP and gene analysis, SCZ and ASD in network analysis, and SCZ only in methylation site analysis. Fisher's exact test was used in the enrichment test. We defined significant enrichment as FDR $<0.05$ and odds ratio $(\mathrm{OR})>1$.

\section{Psychiatric disorder-related signals}

The psychiatric risk gene sets or variants were collected from publications and databases (Table S1). For SNP analysis, we used the latest GWAS results of SCZ [50], ASD [51], and MDD [52]; for CpG analysis, we collected EWAS of SCZ [19, 53]; for gene analysis, we collected the genes from multiple resource which were classified into 36 categories. The gene identifiers were converted to Ensembl Gene IDs in Gencode (GRCh38.p12) using BioMart (https://useast.ensembl.org/index.html).

1. For ASD gene sets, using studies on genetics, differential expression, and co-expression, we examined (1) genes with rare, de novo, loss of function or missense single-nucleotide variants from the NP de novo database [54]; (2) FMRP (Fragile X mental retardation protein) binding targets [55]; (3) candidate genes from the gene reference resource for ASD research database, AutDB [56]; (4) differential expression genes from a recent meta-analysis [57] and the PsychENCODE project [58]; (5) two ASDassociated co-expression modules in postmortem cortex from subjects diagnosed with ASD [59], three ASD-associated co-expression modules from a subsequent RNA-seq study by Gupta et al. [60], and six ASD-associated co-expression modules reported by Parikshak et al. [61].

2. For SCZ gene sets, we examined (1) genes affected by copy number variants (CNVs) [62]; (2) genes identified by linkage and association study [63-65]; (3) genes with de novo variants from NP de novo database [54]; (4) genes identified by convergent functional genomics (CFG) [66]; (5) genes identified by Sherlock integrative analysis [67, 68]; (6) genes identified by Pascal gene-based test [67]; (7) genes expressed differentially in SCZ [57, 58]; and (8) two
SCZ-associated co-expression modules [69].

3. For MDD gene sets, we examined only genes expressed differentially in MDD [57].

\section{Prioritize the sex-differential psychiatric genes}

To prioritize psychiatric candidate genes that are also related to sex bias, we completed a comprehensive integration of the multiple-layers of sex-related genes with the multiple sources of psychiatric-related genes (Fig. 1d). The multiplelayers of sex-related genes contained four types: the DMR genes, DMP-correlated genes, sex-differential expressed genes, and sex-differential network. We identified genes as sex-related psychiatric genes (SRPG) by counting the recurrence of a gene in each category. We developed a generalized score to rank disease related genes, calculated by multiplying the number of times a gene occurred in sexrelated genes categories by the number of times the same gene occurred in related psychiatric disease categories.

\section{Functional enrichment}

R package missMethyl [70], which can adjust for the different number of probes per gene (also called selection bias), was used to identify the functionally enriched pathways for DMPs and DMRs. We used WebGestatle [71] and WebGestalt-KEGG pathway [71] for functional enrichment tests of psychiatric disorder-related genes, and SRPG, respectively. The minimum number of Entrez gene IDs in the category was set to 5 , and the maximum was 2000 . Genome-expressed genes were used as reference. The Benjamini-Hochberg test was used for multiple testing. We defined significant threshold as adjusted $p$ value $<0.05$.

\section{Results}

\section{Sex-differential DNA methylated positions and regions}

We identified 20,450 DMPs significantly associated with sex $($ FDR $<0.05)$ in DLPFC from 166,022 CpG sites (Fig. 2, Fig. S1. Table S2). For the convenience of classification, we named the DMP with higher methylation in females than in males as hypermethylated, and hypomethylated otherwise. Of those 20,450 hits, 75.39\% DMPs were mapped to autosomes, which contained 8693 hypomethylated DMPs $(56.39 \%$ out of the 15,417 DMPs at autosomes). There were $26.50 \%$ DMPs mapped to the X chromosome, which contained 1530 hypomethylated DMPs ( $28.23 \%$ out of the 5419 DMPs at X chromosome).

The DMPs were well-replicated in the two independent replication datasets. In the replicate data of prefrontal 
A

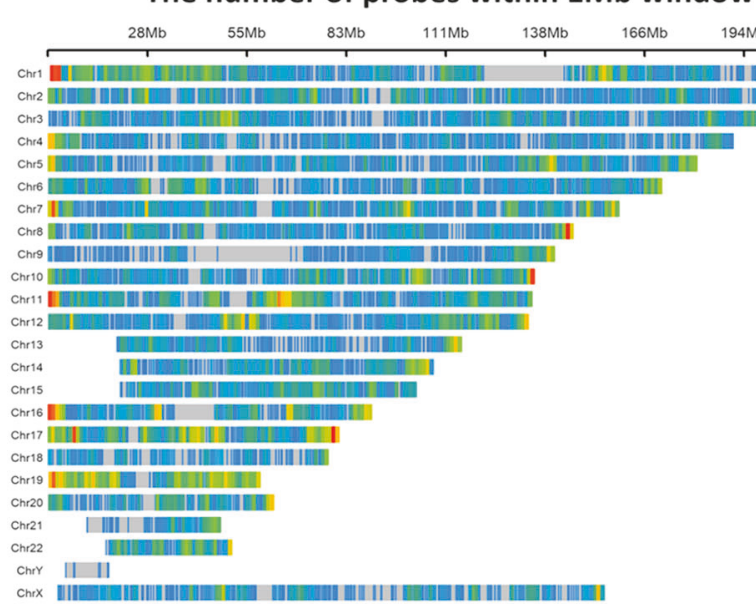

Fig. 2 Significance and difference of sex-differential DNA methylated positions. a Chromosome density plot of sex-differential DNA methylated positions, colored by the $-\log p$ value in $1 \mathrm{MB}$ window

cortex, GSE74193, 86.8\% autosomes DMPs were replicated (FDR < 0.05), 92.8\% X chromosome DMPs were replicated, all of them were consistent in direction with the discovery dataset. In another replicate dataset of multiple brain regions (GSE64509), 72.8\% autosomes DMPs were replicated (FDR < 0.05$)$ and $98.6 \%$ of those replicated had the same direction as the discovery dataset, while $95.9 \% \mathrm{X}$ chromosome DMPs were replicated and all of them had the same direction as the discovery dataset.

There were 2428 sex-differential DMRs mapped to 2513 genes (Table S3), containing 1085 genes with only hypermethylated DMPs (DMR_hyper), 1351 genes with only hypomethylated DMPs (DMR_hypo), and 77 genes with both hypermethylated and hypomethylated DMPs (DMR_both). The DMR genes were strongly enriched for gene sets of neuronal function or potentially related to psychiatric diseases such as axon guide (Benjamini-Hochberg adjusted $p$ value $(\operatorname{adj} . p)=2.04 \mathrm{e}-07)$, MAPK $(\operatorname{adj} . p=2.27 \mathrm{e}-05)$, and calcium signaling (adj. $p=4.95 \mathrm{e}-05)($ Table 1$)$.

\section{Regulatory networks related to sex-differential DNA methylation}

To comprehensively understand the regulatory network of DNA methylation, we considered upstream genetic regulators, downstream gene expression and the PPIs which may be influenced by methylation difference. The genetic regulators were defined by meQTL and the downstream gene-expression analysis was based on the correlation between methylation and gene expression.

For upstream regulation, we used meQTLs to study the relationship between genetic variants (SNP) and DMPs. We started by overlapping the meQTL data of ROSMAP and

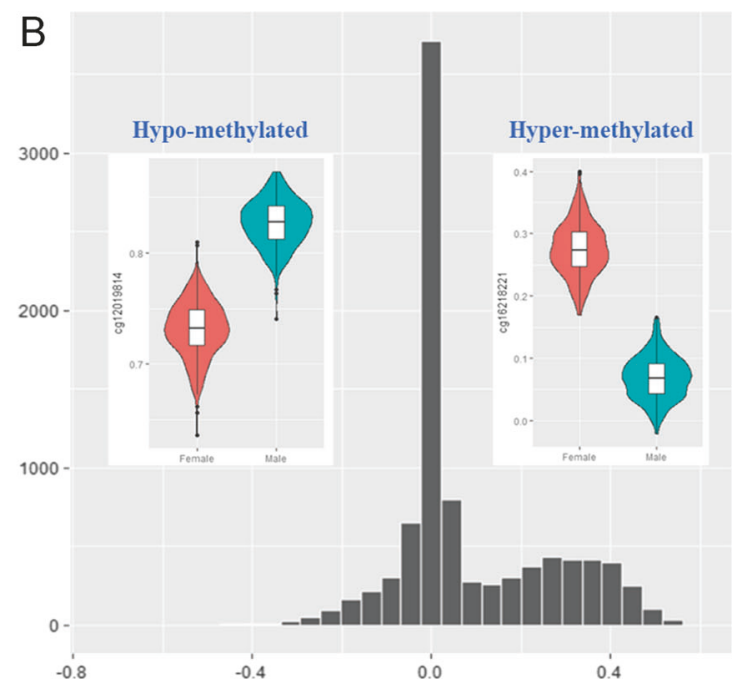

size; $\mathbf{b}$ distribution of the effect size of DMPs (variation between male and female average methyaltion levels). The violin plots shows two DMP examples

Table 1 DMRs mapped genes-enriched KEGG pathways (Top 10)

\begin{tabular}{lll}
\hline Pathway name & \#Genes & adj.p \\
\hline Axon guidance & 24 & $2.04 \mathrm{e}-07$ \\
MAPK signaling pathway & 31 & $2.27 \mathrm{e}-05$ \\
Pathways in cancer & 35 & $2.27 \mathrm{e}-05$ \\
Metabolic pathways & 85 & $2.27 \mathrm{e}-05$ \\
Focal adhesion & 26 & $2.27 \mathrm{e}-05$ \\
Regulation of actin cytoskeleton & 26 & $4.67 \mathrm{e}-05$ \\
Endocytosis & 25 & $4.67 \mathrm{e}-05$ \\
Calcium signaling pathway & 23 & $4.95 \mathrm{e}-05$ \\
Amoebiasis & 16 & $2.00 \mathrm{e}-04$ \\
Apoptosis & 14 & $3.00 \mathrm{e}-04$ \\
\hline
\end{tabular}

Adj.P Benjamini-Hochberg adjusted $p$ value

GSE74193 to obtain a list of meQTL with good reproducibility, which included 434,312 meQTL pairs $(253,471$ SNPs and 45,049 CpGs) that were significant (with FDR $<$ 0.05 ) in both dataset. From the reproducible meQTLs, we found 22,782 sex-related meQTLs (SNP-DMP pairs) that included 2644 DMPs (12.9 \% of the 20,450 DMPs) associated with 18,349 SNPs (Table S4). These results indicated that $12.9 \%$ DMPs were regulated by genetic variants.

For target gene expression, we performed correlation analysis between methylation and gene expression in data of DLPFC from the ROSMAP. The correlation test of 20,450 DMP with nearby genes' expression $(10 \mathrm{~kb})$ showed that 1363 DMPs had a significant correlation with 627 genes $($ FDR $<0.05)$, forming 1525 DMP-gene pairs (Table S5). These results showed that 6.7\% DMPs may influenced the gene expression. 
We further used the DMP as a linker between SNPs and genes, and built 3161 SNP-DMP-gene groups, containing 2054 SNPs, 276 DMPs, and 200 genes (Table S6). These SNP-DMP-gene groups connected the genetic variants to gene expression through sex-differential DNA methylation. For example, rs10143703 can regulate cg04842215, and methylation of cg04842215 correlated with expression of CBLN3 (Fig. 3a).
We further extended regulatory network of DNA methylation by adding PPIs. Expression of many genes may not be influenced by sex directly, but they interact with differently expressed genes to execute their specific functions. To retrieve these related genes, we obtained 19 PPI subnetworks (Fig. 3b, Fig. S2) that were related to our sexdifferential methylated DNA. For example, promoters of FOXO4, FTL, BRF2, GREB3L3, and TBCB in these
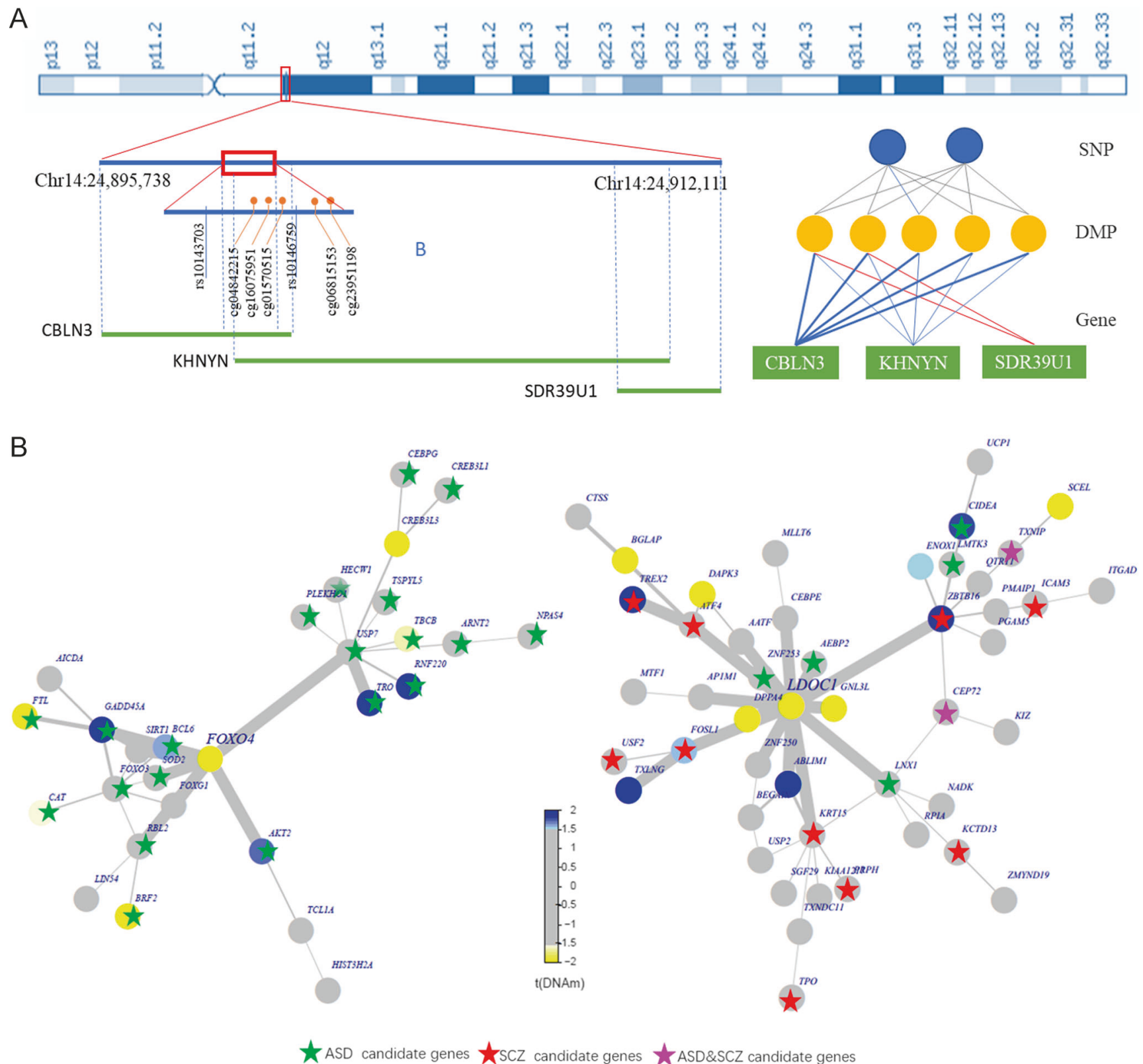

Fig. 3 Sex-differential regulatory network. a Example of SNP-DMPGene groups. There are $12 \mathrm{SNP}-\mathrm{DMP}-\mathrm{Gene}$ groups in this region on Chromosome 14: 24,895,387-24,912,111, involving two SNPs, five DMPs and three genes. The diagram shows the location of them while the cartoon diagram shows their relationship. The gray line represents meQTLs with FDR $<0.05$. The blue lines represent negative correlation and red lines represent positive correlations. b Example of sexdifferential PPI subnetworks. Every node represents a gene. The color of nodes represents differential methylation levels in corresponding promoters (Yellow: hypermethylated in the female; Blue: hypomethylated in female). The edges were built based on the proteinprotein interaction in Pathway Common. The width of the edge is the estimation of effect sizes. Stars represent the candidate genes (Green: ASD candidate genes, Red: SCZ candidate genes, Purple: both ASD and SCZ candidate genes) 
subnetworks exhibited hypermethylation in females (Fig. 3b). In contrast, GADD45A, AKT2, TRO, and RNF22O exhibited hypomethylation in females. Many genes in these subnetworks did not show sex difference, such as $C E B P G$, CREB3L1, HECW1, TSPYL5, PLEKHO1, USP7, ARNT2, NPAS4, CAT, FOXO3, FOXG1, RBL. Through the interaction with the genes showed sex-biased methylation, these genes who did not show sex difference may function in a sex different way.

\section{Overrepresentation of psychiatric disease signals in sex-differential loci}

To learn whether psychiatric disorder-related genes show sex-differential methylation or regulation, we tested for enrichment between the signals related to sex-differential methylation networks and genetic signals associated with psychiatric disorders. Focusing on SCZ, ASD, and MDD, we found significant enrichment at the SNP, methylation site, gene, and network levels.

At the SNP level, we compared the SNPs which regulated DMPs by meQTLs with the GWAS SNPs which associated with SCZ, ASD and MDD. We extracted 9138 SNPs associated with SCZ at $p<5.00 \mathrm{e}-08$ from PGC [72] and found 63 SNPs that regulated DMPs. These SNPs were more likely to regulate DMPs (63 of 9138 compared with the background 18,349 of $8,379,106$, Odds ratio for enrichment $(\mathrm{OR})=3.15, p=8.47 \mathrm{e}-15)$. For ASD, 93 SNPs were extracted at $p<5.00 \mathrm{e}-08$ from PGC [51], but none of them regulated DMPs. For MDD, we extracted 912 SNPs associated with MDD at $p<5.00 \mathrm{e}-08$. We did not observe enrichment of MDD-associated SNPs in those that regulate DMPs. However, one SNP, rs61990288, which was associated with MDD, was also a meQTL SNP that regulated a DMP.

At the CpG level, we compared our sex-related DMPs with the EWAS results of Jaffe et al. [19] $(n=750$ samples), who tested SCZ brains. Using an EWAS $p$ value less than $5.00 \mathrm{e}-05$ as the cut-off, we extracted $1059 \mathrm{CpG}$ loci associated with SCZ. Among these $1059 \mathrm{CpG}$ sites, 21 CpGs that was associated with SCZ and show sexdifferential methylation. We did not find enrichment of sex-differential DMPs $(\mathrm{OR}=0.16, p=1.68 \mathrm{e}-35)$. We also used SCZ EWAS results from Hannon et al. [53], who quantified DNA methylation from blood samples. The DMPs were not enriched for CpGs associated with SCZ $(\mathrm{OR}=0.01$, enrichment $p=4.39 \mathrm{e}-05)$, but $81 \mathrm{CpGs}$ was associated with SCZ and show sex-differential methylation.

To determine whether genes associated with ASD, SCZ, and MDD show sex-related differential methylation, we collected disease candidate genes that covered genetics, differential expression, and co-expression studies (Fig. 4a, Table S7). For ASD-related gene analysis, we observed significant enrichment of DMR genes with ASD-related risk genes with loss of function de novo variants $(\mathrm{OR}=1.77, p=5.44 \mathrm{e}-3)$, FMRP gene set $(\mathrm{OR}=1.71, p=3.00 \mathrm{e}-9)$ and candidate genes from AutDB $(\mathrm{OR}=2.86, p=2.84 \mathrm{e}-10)$, but genes with missense de novo mutations were not enriched in DMR genes $(\mathrm{OR}=1.34, p=5.37 \mathrm{e}-2)$. DMR genes were also enriched in ASD-related differentially expressed gene sets and co-expression modules. For SCZ-related gene analysis, we observed significant enrichment of DMR genes with missense de novo mutation genes, loss of function de novo mutation genes, differentially expressed SCZ genes, and also SCZ-associated co-expression genes. However, in contrast with ASD, the DMR genes were not enriched in genes identified by linkage [63-65], Sherlock [67, 68], Pascal [67], and CFG [66] in SCZ. For MDD, we did not find enrichment of DMR genes with differentially expressed or co-expressed genes.

To take the direction of the DMR into account, we tested for enrichment of disease-related genes in the DMR_hyper genes, DMR_hypo genes and DMR_both genes (Fig. 4b, Table S7). We found upregulated different expression gene sets and upregulated co-expression gene sets in ASD were enriched in DMR_hyper genes, whereas downregulated different expression gene sets and downregulated coexpression genes sets in ASD were enriched in DMR_hypo genes. In contrast, in gene sets of MDD, we found downregulated differential expression genes enriched in DMR_hyper genes.

We also tested the enrichment of DMP-correlated expression genes and sex-differential expressed genes (Table S7, Fig. 4a) against genes associated with ASD, SCZ, and MDD. By clustering analysis, we found DMPcorrelated genes had a similar enrichment pattern as DMR genes, but not the sex-differential expressed genes.

While some sex-differential methylated genes are also risk genes, some other risk genes may interact with sexdifferential genes through protein networks. Differential methylation can impact functions of both types of genes at the system level. The ASD- and SCZ-related genes were mapped to the PPI subnetworks that exhibit sex-related differential DNA methylation. The sex-differential PPI subnetworks connected the sexdifferential genes to psychiatric disorder candidate genes. For example, the sex-differential network-with FOXO4 as a hub gene, a sex-differential gene, interacts with 29 other genes and 20 of them were ASD-related genes (Fig. 3b). Even though they were not sexdifferential methylated genes, their functions were affected by their sex-dependent patterns. 


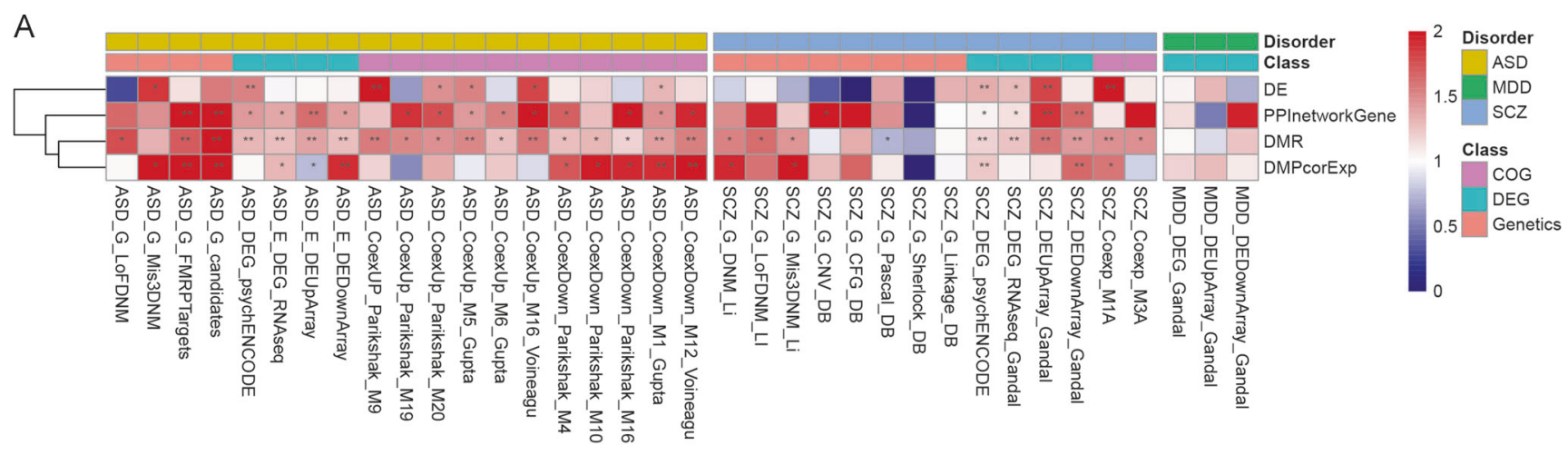

B

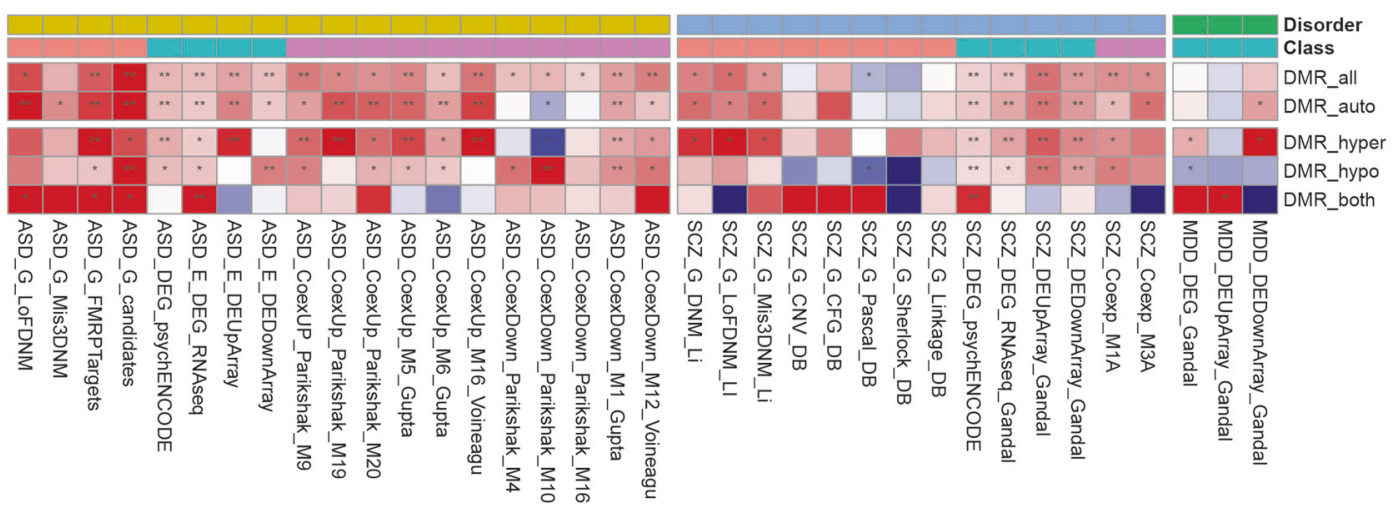

Fig. 4 A compressive overrepresentation of psychiatric candidate gene sets in sex-biased genes. a Overrepresentation of psychiatric candidate gene sets in DMR genes, DMP-correlated expressed genes, differentially expressed genes, and PPI network genes, clustered by the enrichment value. b Overrepresentation of psychiatric candidate gene sets in DMR genes and subset of DMR genes. The $x$-axis shows 34

\section{Prioritize the psychiatric risk genes that involve sex bias}

Since enrichment of sex-related genes was observed among psychiatric disorder-associated genes, we attempted to identify specific risk genes that are under sex-dependent regulation. We defined SRPGs as genes that were associated with sex at least once and associated with at least one of the psychiatric disorders (SCZ, MDD, or ASD) (Table S10). For example, complexin/synaphin gene, CPLX1 was a DMR gene, and its expression level correlated with a DMP. CPLXI was related with ASD and SCZ from multiple studies involving genetic variants [55] and co-expression changes in postmortem brain of ASD patients [57, 59, 60]. Therefore, $C P L X 1$ was a SRPG.

Of the 13,055 studied genes, we identified 2080 SRPGs (1498 ASD-related, 1349 SCZ-related, and 51 MDD-related). These genes were subgroup of psychiatric disorder genes which enriched in synapse and signaling pathways (Table 2, Table S11). Of the 1498 SPRGs related to ASD, 98 genes were associated with sex-differential features and ASD-associated features in several analyses. The top ten ranking of SPRGs for ASD were CPLX1, HEBP2, SYP, gene sets divided based on the psychiatric disorder and labeled by type; the $y$-axis shows the DMR genes, DMP-correlated expressed genes and differentially expressed genes. The color of the box shows the odds ratio for enrichment (red for enrichment, blue for deletion). “*” indicates enrichment is statistically significant $(p<0.05)$, “**” indicates $p<0.001$

Table 2 Prioritized genes enriched KEGG pathway

\begin{tabular}{lll}
\hline Pathway name & \#Genes & adj. $p$ \\
\hline Dopaminergic synapse & 16 & $2.29 \mathrm{e}-04$ \\
Glutamatergic synapse & 13 & $2.99 \mathrm{e}-03$ \\
cAMP signaling pathway & 17 & $5.33 \mathrm{e}-03$ \\
Retrograde endocannabinoid signaling & 11 & $1.00 \mathrm{e}-02$ \\
Circadian entrainment & 10 & $2.34 \mathrm{e}-02$ \\
Amphetamine addiction & 8 & $2.77 \mathrm{e}-02$ \\
Calcium signaling pathway & 14 & $2.77 \mathrm{e}-02$ \\
Chagas disease (American trypanosomiasis) & 10 & $2.77 \mathrm{e}-02$ \\
GABAergic synapse & 9 & $2.89 \mathrm{e}-02$ \\
MAPK signaling pathway & 17 & $2.89 \mathrm{e}-02$ \\
Serotonergic synapse & 10 & $3.80 \mathrm{e}-02$ \\
FoxO signaling pathway & 11 & $3.80 \mathrm{e}-02$ \\
Alcoholism & 13 & $4.35 \mathrm{e}-02$ \\
AGE-RAGE signaling pathway in diabetic & 9 & $4.96 \mathrm{e}-02$ \\
complications & & \\
\hline
\end{tabular}

Adj.p Benjamini-Hochberg adjusted $p$ value

CD99L2, ZC3HAV1, SAT1, HECW1, TRO, CD40, STS, and NRXN3. Among the 1349 SCZ-related SRPGs, 55 genes 
were supported by multiple lines of disease risk and differential methylation data. The genes ANOS1, MAGI2, CHRDL1, GNG12, MSL3, SMC1A, ITM2A, PLS3, CDK16, ZC3HAVI, and UBTF were ranked in the top ten. Eight genes (AR, WWC3, NOS1, PAX8, GRB7, SYTL1, CLIC6, $B E G A I N$ ) of the MDD-related SRPGs were supported by multiple data. Functional enrichment tests showed that the SPRGS with more than two associations with psychiatric disease and sexual differences $(n=653)$ were enriched in synapse-related pathways like dopaminergic synapse $(\operatorname{adj} . p=2.3 \mathrm{e}-4)$, glutamatergic synapse $(\operatorname{adj} . p=2.9 \mathrm{e}-4)$, GABAergic synapse (adj. $p=2.9 \mathrm{e}-2$ ), and serotonergic synapse (adj. $p=3.8 \mathrm{e}-2$ ). These SPRGs were also enriched in signaling pathways such as the cAMP (adj. $p=5.3 \mathrm{e}-3)$, calcium (adj. $p=2.8 \mathrm{e}-2)$, MAPK (adj. $p=2.9 \mathrm{e}-2)$, and FoxO (adj. $p=3.8 \mathrm{e}-2)$ (Table 2$)$.

\section{Discussion}

We identified sex-differential DNA methylation and regulatory networks in one of the largest studies of postmortem human brain tissue to date. Thousands of sex-differential DMPs and DMRs were identified and replicated. Regulatory networks that connect the DMPs with SNPs, gene expression, and PPI were built up. Enrichment of psychiatric disease-associated genes in DMPs, DMRs, and networks was detected.

To assess the consistency between our findings and prior results on sex-differential DNA methylation, we compared DMPs in the current analysis with five relevant publications (Table 3). These studies differed from ours either in DNA methylation analysis platform $(27 \mathrm{~K}$ in McCarthy et al. [26]), tissue types (cord blood in Yousefi et al. [20] and whole blood in Singmann et al. [22]), or subjects' age range (fetal brain in Spiers et al.). The sample size in the current study was much larger than in previous studies. Our results replicated from 10.9 to $45.3 \%$ of the probes that passed QC. Totally, $68.4 \%$ of our DMPs results are novel findings. These novel findings were based on our strict criteria that contained only the high-quality probes [39] and controlled for potential artifacts such as batch effects, position effects, and cell-type component.

Our data show that sex-differential genes are enriched in pathways known to be important in neurons including axon guidance, MAPK signaling, and calcium signaling. These pathways have been previously suggested as being involved in psychiatric risks. For example, axon guidance pathways strongly influence human speech and language, and deficits in language and communication are hallmarks of ASD [73]. The MAPK singling pathway is reported to determine depression-like behavior and anxiety [74], which may be contribute to the different prevalence between males and females for MDD. Calcium signaling pathways regulate many neural functions involving the generation of brain rhythms, information processing and the changes in synaptic plasticity [75]. Dysregulation of calcium signaling pathways has been implicated in the development of psychiatric diseases such as SCZ [75]. The discovery that sexdifferential genes are enriched in these important pathways may help us to better understand the sex-related mechanisms underlying psychiatric disorders.

Our study curated a regulatory system related to sexdifferential DNA methylation, which supports our first hypothesis that sex-differences exists in both DNA methylation and its regulatory network. For each of the DMPs, putative upstream genetic regulators and downstream target genes were identified by connecting DMPs with meQTL, genes, and PPI. Therefore, a more complete biological system that either contributes to or is affected by sexdifferences come together for their potential involvement to disease risks.

We conducted a comprehensive comparison between the sex-differential methylation regulation system and psychiatric disorder risk factors, providing support for our second hypothesis that psychiatric disorder-related genes have different methylation levels between males and females. We took advantage of numerous types of data including GWAS, rare variants studies, EWAS, differential expression and coexpression studies to capture different aspects of genetic, environmental or the genetic-environmental interaction effects and provide new insights into the disease etiology. We found different methylation regulation systems between male and female enriched in these different types of psychiatric risk factors.

We found common variants that regulate DMPs were enriched in SCZ GWAS signals, which expands the multiple liability model. The multiple liability model assumes the same genetic variants have the same effect on males and females. Our study demonstrated that the same genetic variants have different influences on males and females in DNA methylation. For example, GWAS signals of SCZ such as rs4702 and rs12332385, can regulate sex-related DMPs through meQTL. Therefore, despite only calculating the accumulation of risk alleles in the multiple liability model, both the number of risk alleles and the effect size should be included in the model to explain the sex-bias feature of the disorders. Beside the common variants from GWAS studies, we observed that sex-differential DMR genes were enriched in de novo mutation genes related to SCZ and ASD, which provides evidence that the rare variant genes contribute to SCZ and ASD and show a sexdifferential methylated pattern in DLPFC. We identified EWAS signals showing sex-differential methylation, suggesting the baseline methylation level of these EWAS signals of SCZ is different between males and females. In the 
Table 3 Comparison of DMPs in autosome with other published studies

\begin{tabular}{|c|c|c|c|c|c|c|c|}
\hline Study & Sample size & Population & Tissue & Platform & $\begin{array}{l}\text { \# Of } \\
\text { AUDMPS }\end{array}$ & $\begin{array}{l}\text { \# Of AUDMPS } \\
\text { in PQC }\end{array}$ & $\begin{array}{l}\text { \# Of AUDMPS } \\
\text { Replicated (\%) }\end{array}$ \\
\hline McCarthy et al. [26] & 6795 & - & Multiple types & $27 \mathrm{~K}$ & 235 & 75 & $34(45.3 \%)$ \\
\hline $\mathrm{Xu}$ et al. [25] & 46 & Caucasian & Prefrontal cortex & $450 \mathrm{~K}$ & 614 & 266 & $79(29.7 \%)$ \\
\hline Spiers et al. [21] & 179 & Caucasian & Fetal brain & $450 \mathrm{~K}$ & 525 & 223 & $85(36.5 \%)$ \\
\hline Yousefi et al. [20] & 111 & $\begin{array}{l}\text { Mexican- } \\
\text { American }\end{array}$ & $\begin{array}{l}\text { Umbilical cord } \\
\text { blood }\end{array}$ & $450 \mathrm{~K}$ & 3031 & 1236 & $301(24.3 \%)$ \\
\hline Singmann et al. [22] & 1799 & Caucasian & Whole blood & $450 \mathrm{~K}$ & 1178 & 512 & $56(10.9 \%)$ \\
\hline
\end{tabular}

downstream genes, we found DMP-correlated genes enriched in candidate genes of ASD, SCZ, and MDD. For example, we found significant enrichment of differentially expressed genes in MDD enriched in sex-related DMR_hyper genes. Four CpG sites (cg22466678, cg15296664, and cg08802841 at intergenic region, cg20722088 at $3^{\prime}$ UTR) on DUSP6 genes show hypomethylation in female. The DUSP6 has been reported to be a female-specific hub gene which influenced stress susceptibility in females [76].

One of the most interesting findings in our study is from the comparison of sex-differential methylation and expression results with ASD risk genes. We observed enrichment of sex-differential methylation (DMR genes and DMPcorrelated genes) in both ASD-risk genes and ASD-related pathways, suggesting the ASD-risk genes may contribute to the sex difference of the disease through DNA methylation but not gene expression. This result expanded the results of Werling et al. [6], who reported that an ASD-related pathway, but not the ASD-risk genes, were enriched in sexdifferential expressed genes. ASD-related differentially expressed genes and co-expressed genes, not the ASD-risk genes that had genetic variants, were enriched in sexdifferential expressed genes in the current study. However, we observed enrichment of sex-differential methylation (DMR genes and DMP-correlated genes) in both ASD-risk genes and ASD-related pathways. One of the possible explanations is that the DNA methylation as the upstream regulator is more sensitive than gene expression [32]. We found the DMP-correlated genes are more likely to be differentially expressed, which provided an in-directed support to the explanation. Therefore, comprehensive analyses that combine methylation and gene expression are crucial to reach a better understanding of complex diseases and their sex differences.

We found ASD loss of function de novo genes are enriched for DMR genes, while genes with missense de novo mutation are not enriched for DMR genes. Although both loss of function de novo genes and missense de novo mutation are associated with ASD, these different enrichment result remain us to consider the genic intolerance. Genic intolerance is a quantitative assessment of how well genes tolerate functional genetic variation on a genome-wide scale [77]. Genes with de novo mutations in ASD are generally intolerant genes, having important function. It is possible that methylation levels are strictly regulated in such intolerant genes, which result in smaller variation across individuals and cause the statistically significant sex difference. Through the gene intolerant analysis, we compared the residual variation intolerance score (RVIS) of DMR genes and non-DMR genes, and we found the DMR genes have a significant lower mean value of RVIS score (mean DMR $=-0.13$, mean non-DMR $=0.01$, $p=1.754 \mathrm{e}-10)$, which means the DMR genes are intolerant genes with important functions (Supplementary method).

Most notably, we found upregulated genes in ASD were enriched in hypermethylated DMR genes in females. Hypermethylation may result in low-gene expression level, which means compare to males, females have a lower expression level of these upregulated genes in ASD. In other words, the relative amount of gene change required for female to reach ASD diagnosis is larger than males, which can explain the different prevalence between male and female. In the contrast, the downregulated genes in MDD were enriched in hypermethylated genes in MDD, which means for the females the relative change is smaller than males to reach the MDD diagnosis. These results provide compelling evidence for the multifactorial model which hypothesis the sex-specific genetic and environmental factors in the sex with lower incidence shift its' total liability distribution away from the diagnostic threshold.

We prioritized psychiatric genes related to sex-bias and highlighted some important pathways which were sexdifferential and related to psychiatric disorders, including important psychiatric disease candidate genes like $N R X N 1$, NRXN2, NRXN3, PDE4A, SHANK2. Our study suggests that the synapse-related pathway and several signaling pathways differ by sex and may be disrupted in psychiatric disorders. For example, dopaminergic, glutamatergic, and GABAergic synapse, all suspected of being involved in psychiatric disorders, all differ between male and female. Studies targeting these genes and pathways should take sex 
into account in design and analysis. Studies of these genes and pathways may reveal the biology that drives sex-related features of the disorders.

The current study has several limitations. DNA methylation exhibits spatiotemporal patterns that cannot be fully captured. Our analyses utilized gene methylation and expression data from the human adult prefrontal cortex. Other brain regions known to be robustly sex-differential were not represented in our data sets, such as the hypothalamic nuclei. The current study used bulk tissues, not specific cell types, and expression of genes related to psychiatric disorders may vary among brain cell types. Celltype specific studies based on the single-cell or deconvolutional data should be explored in the future. The majority of our discovery samples were from an older population that was post-reproductive age. This age range does not coincide with the typical age of onset for the major psychiatric disorders. Samples from children, adolescent, young adults need to be explored in the future.

Acknowledgements We thank Barry Knox for revising; the anonymous reviewers for helpful comments.

Funding This work was supported by NIH grants 1U01MH10334001, 1R01ES024988 (to C. Liu) and National Natural Science Foundation of China grants 81401114 and 31571312 , the National Key Plan for Scientific Research and Development of China (2016YFC1306000), Innovation-Driven Project of Central South University (Nos. 2015CXS034 and 2018CX033) (to C. Chen). We also sincerely thank Chicago Biomedical Consortium with support from the Searle Funds at The Chicago Community Trust.

Author contributions C.C. and C.L. designed the study and interpreted the results. Y.X. performed the data processing, different methylation and network analysis, overrepresentation analysis, and prioritize the analysis. D.J. ran the cell component deconvolution. C.Z. initiated and piloted the study. K.W. and C.J. ran to the data quality control of GSE74193 dataset and correlation analysis of methylation and gene expression. Y.X., H.L., and J.X. collected the data and ran the functional enrichment analysis. Y.C. collected the psychiatricrelated gene lists. Y.J. run the gene intolerant analysis. Y.X. drafted the paper with assistance from C.C., C.L., R.K., and G.G.

\section{Compliance with ethical standards}

Conflict of interest The authors declare that they have no conflict of interest.

Publisher's note: Springer Nature remains neutral with regard to jurisdictional claims in published maps and institutional affiliations.

\section{References}

1. Werling DM, Geschwind DH. Sex differences in autism spectrum disorders. Curr Opin Neurol. 2013;26:146-53.

2. Christensen DL, Baio J, Van Naarden Braun K, Bilder D, Charles J, Constantino JN, et al. Prevalence and characteristics of autism spectrum disorder among children aged 8 years-autism and developmental disabilities monitoring network, 11 sites,
United States, 2012. Morb Mortal Wkly Rep Surveill Summ. 2016;65:1-23.

3. Abel KM, Drake R, Goldstein JM. Sex differences in schizophrenia. Int Rev Psychiatry. 2010;22:417-28.

4. Kessler RC, Chiu WT, Demler O, Merikangas KR, Walters EE. Prevalence, severity, and comorbidity of 12-month DSM-IV disorders in the National Comorbidity Survey Replication. Arch Gen Psychiatry. 2005;62:617-27.

5. Kornstein SG, Schatzberg AF, Thase ME, Yonkers KA, McCullough JP, Keitner GI, et al. Gender differences in chronic major and double depression. J Affect Disord. 2000;60:1-11.

6. Werling DM, Parikshak NN, Geschwind DH. Gene expression in human brain implicates sexually dimorphic pathways in autism spectrum disorders. Nat Commun. 2016;7:10717.

7. Mitra I, Tsang K, Ladd-Acosta C, Croen LA, Aldinger KA, Hendren RL, et al. Pleiotropic mechanisms indicated for sex differences in autism. PLoS Genet. 2016;12:e1006425.

8. Khramtsova EA, Davis LK, Stranger BE. The role of sex in the genomics of human complex traits. Nat Rev Genet. 2018; 20:173-90.

9. Hong DS, Reiss AL. Cognitive and neurological aspects of sex chromosome aneuploidies. Lancet Neurol. 2014;13:306-18.

10. de Castro-Catala M, Barrantes-Vidal N, Sheinbaum T, MorenoFortuny A, Kwapil TR, Rosa A. COMT-by-sex interaction effect on psychosis proneness. Biomed Res Int. 2015;2015:829237.

11. Vink JM, Bartels M, van Beijsterveldt TC, van Dongen J, van Beek JH, Distel MA, et al. Sex differences in genetic architecture of complex phenotypes? PLoS ONE. 2012;7:e47371.

12. Traglia M, Bseiso D, Gusev A, Adviento B, Park DS, Mefford JA, et al. Genetic mechanisms leading to sex differences across common diseases and anthropometric traits. Genetics. 2017;205: 979-92.

13. Kubota T, Miyake K, Hirasawa T. Epigenetic understanding of gene-environment interactions in psychiatric disorders: a new concept of clinical genetics. Clin Epigenetics. 2012;4:1.

14. Lev Maor G, Yearim A, Ast G. The alternative role of DNA methylation in splicing regulation. Trends Genet. 2015; 31:274-80.

15. Moore LD, Le T, Fan G. DNA methylation and its basic function. Neuropsychopharmacology. 2013;38:23-38.

16. Bell JT, Pai AA, Pickrell JK, Gaffney DJ, Pique-Regi R, Degner $\mathrm{JF}$, et al. DNA methylation patterns associate with genetic and gene expression variation in HapMap cell lines. Genome Biol. 2011;12:R10.

17. Maschietto M, Bastos LC, Tahira AC, Bastos EP, Euclydes VLV, Brentani A, et al. Sex differences in DNA methylation of the cord blood are related to sex-bias psychiatric diseases. Sci Rep. 2017;7:44547.

18. Yuan Y, Liu L, Chen H, Wang Y, Xu Y, Mao H, et al. Comprehensive characterization of molecular differences in cancer between male and female patients. Cancer Cell. 2016;29:711-22.

19. Jaffe AE, Gao Y, Deep-Soboslay A, Tao R, Hyde TM, Weinberger DR, et al. Mapping DNA methylation across development, genotype and schizophrenia in the human frontal cortex. Nat Neurosci. 2016;19:40-47.

20. Yousefi P, Huen K, Dave V, Barcellos L, Eskenazi B, Holland N. Sex differences in DNA methylation assessed by 450 K BeadChip in newborns. BMC Genomics. 2015;16:911.

21. Spiers H, Hannon E, Schalkwyk LC, Smith R, Wong CC, O'Donovan MC, et al. Methylomic trajectories across human fetal brain development. Genome Res. 2015;25:338-52.

22. Singmann P, Shem-Tov D, Wahl S, Grallert H, Fiorito G, Shin $\mathrm{SY}$, et al. Characterization of whole-genome autosomal differences of DNA methylation between men and women. Epigenetics Chromatin. 2015;8:43. 
23. Nugent BM, Wright CL, Shetty AC, Hodes GE, Lenz KM, Mahurkar A, et al. Brain feminization requires active repression of masculinization via DNA methylation. Nat Neurosci. 2015;18:690-7.

24. Loke H, Harley V, Lee J. Biological factors underlying sex differences in neurological disorders. Int $\mathrm{J}$ Biochem Cell Biol. 2015;65:139-50.

25. Xu H, Wang F, Liu Y, Yu Y, Gelernter J, Zhang H. Sex-biased methylome and transcriptome in human prefrontal cortex. Hum Mol Genet. 2014;23:1260-70.

26. McCarthy NS, Melton PE, Cadby G, Yazar S, Franchina M, Moses EK, et al. Meta-analysis of human methylation data for evidence of sex-specific autosomal patterns. BMC Genomics. 2014;15:981.

27. Hall E, Volkov P, Dayeh T, Esguerra JLS, Salö S, Eliasson L, et al. Sex differences in the genome-wide DNA methylation pattern and impact on gene expression, microRNA levels and insulin secretion in human pancreatic islets. Genome Biol. 2014;15:522.

28. Uddin M, Sipahi L, Li J, Koenen KC. Sex differences in DNA methylation may contribute to risk of PTSD and depression: a review of existing evidence. Depress Anxiety. 2013;30:1151-60.

29. Qureshi IA, Mehler MF. Genetic and epigenetic underpinnings of sex differences in the brain and in neurological and psychiatric disease susceptibility. Prog Brain Res. 2010;186:77-95.

30. De Jager PL, Srivastava G, Lunnon K, Burgess J, Schalkwyk LC, $\mathrm{Yu}$ L, et al. Alzheimer's disease: early alterations in brain DNA methylation at ANK1, BIN1, RHBDF2 and other loci. Nat Neurosci. 2014;17:1156-63.

31. Horvath S, Mah V, Lu AT, Woo JS, Choi OW, Jasinska AJ, et al. The cerebellum ages slowly according to the epigenetic clock. Aging. 2015;7:294-306.

32. Ng B, White CC, Klein HU, Sieberts SK, McCabe C, Patrick E, et al. An XQTL map integrates the genetic architecture of the human brain's transcriptome and epigenome. Nat Neurosci. 2017;20:1418-26.

33. Cerami EG, Gross BE, Demir E, Rodchenkov I, Babur O, Anwar N, et al. Pathway Commons, a web resource for biological pathway data. Nucleic Acids Res. 2011;39(Database issue):D685-690.

34. West J, Beck S, Wang X, Teschendorff AE. An integrative network algorithm identifies age-associated differential methylation interactome hotspots targeting stem-cell differentiation pathways. Sci Rep. 2013;3:1630.

35. Morris TJ, Butcher LM, Feber A, Teschendorff AE, Chakravarthy AR, Wojdacz TK, et al. ChAMP: 450k chip analysis methylation pipeline. Bioinformatics. 2014;30:428-30.

36. Zhou W, Laird PW, Shen H. Comprehensive characterization, annotation and innovative use of Infinium DNA methylation BeadChip probes. Nucleic Acids Res. 2017;45:e22.

37. Troyanskaya O, Cantor M, Sherlock G, Brown P, Hastie T, Tibshirani R, et al. Missing value estimation methods for DNA microarrays. Bioinformatics. 2001;17:520-5.

38. Dedeurwaerder S, Defrance M, Calonne E, Denis H, Sotiriou C, Fuks F. Evaluation of the infinium methylation 450K technology. Epigenomics. 2011;3:771-84.

39. Naeem H, Wong NC, Chatterton Z, Hong MK, Pedersen JS, Corcoran NM, et al. Reducing the risk of false discovery enabling identification of biologically significant genome-wide methylation status using the HumanMethylation450 array. BMC Genomics. 2014;15:51.

40. Houseman EA, Accomando WP, Koestler DC, Christensen BC, Marsit CJ, Nelson HH, et al. DNA methylation arrays as surrogate measures of cell mixture distribution. BMC Bioinformatics. 2012;13:86

41. Guintivano J, Aryee MJ, Kaminsky ZA. A cell epigenotype specific model for the correction of brain cellular heterogeneity bias and its application to age, brain region and major depression. Epigenetics. 2013;8:290-302.

42. Teschendorff AE, Menon U, Gentry-Maharaj A, Ramus SJ, Gayther SA, Apostolidou S, et al. An epigenetic signature in peripheral blood predicts active ovarian cancer. PLoS ONE. 2009;4:e8274.

43. Chen C, Grennan K, Badner J, Zhang D, Gershon E, Jin L, et al. Removing batch effects in analysis of expression microarray data: an evaluation of six batch adjustment methods. PLOS ONE. 2011;6:e17238.

44. Leek JT, Storey JD. Capturing heterogeneity in gene expression studies by surrogate variable analysis. PLoS Genet. 2007;3: 1724-35.

45. Jiao C, Zhang C, Dai R, Xia Y, Wang K, Giase G, et al. Positional effects revealed in Illumina methylation array and the impact on analysis. Epigenomics. 2018;10:643-59.

46. Du P, Zhang X, Huang CC, Jafari N, Kibbe WA, Hou L, et al. Comparison of Beta-value and $\mathrm{M}$-value methods for quantifying methylation levels by microarray analysis. BMC Bioinformatics. 2010;11:587.

47. Ritchie ME, Phipson B, Wu D, Hu Y, Law CW, Shi W, et al. limma powers differential expression analyses for RNAsequencing and microarray studies. Nucleic Acids Res. 2015;43: e47.

48. Peters TJ, Buckley MJ, Statham AL, Pidsley R, Samaras K, VL R, et al. De novo identification of differentially methylated regions in the human genome. Epigenetics Chromatin. 2015;8:6.

49. Jiao Y, Widschwendter M, Teschendorff AE. A systems-level integrative framework for genome-wide DNA methylation and gene expression data identifies differential gene expression modules under epigenetic control. Bioinformatics. 2014;30:2360-6.

50. Schizophrenia Working Group of the Psychiatric Genomics C. Biological insights from 108 schizophrenia-associated genetic loci. Nature. 2014;511:421-7.

51. Grove J, Ripke S, Als TD, Mattheisen M, Walters R, Won H, et al. Common risk variants identified in autism spectrum disorder. Nat Genet. 2017;51:224774.

52. Major Depressive Disorder Working Group of the Psychiatric GC, Ripke S, Wray NR, Lewis CM, Hamilton SP, Weissman MM, et al. A mega-analysis of genome-wide association studies for major depressive disorder. Mol Psychiatry. 2013;18:497-511.

53. Hannon E, Dempster E, Viana J, Burrage J, Smith AR, Macdonald $\mathrm{R}$, et al. An integrated genetic-epigenetic analysis of schizophrenia: evidence for co-localization of genetic associations and differential DNA methylation. Genome Biol. 2016;17:176.

54. Li J, Cai T, Jiang Y, Chen H, He X, Chen C, et al. Genes with de novo mutations are shared by four neuropsychiatric disorders discovered from NPdenovo database. Mol Psychiatry. 2016;21:298

55. Darnell JC, Van Driesche SJ, Zhang C, Hung KY, Mele A, Fraser $\mathrm{CE}$, et al. FMRP stalls ribosomal translocation on mRNAs linked to synaptic function and autism. Cell. 2011;146:247-61.

56. Basu SN, Kollu R, Banerjee-Basu S. AutDB: a gene reference resource for autism research. Nucleic Acids Res. 2009;37(Database issue):D832-836.

57. Gandal MJ, Haney JR, Parikshak NN, Leppa V, Ramaswami G, Hartl C, et al. Shared molecular neuropathology across major psychiatric disorders parallels polygenic overlap. Science. 2018;359:693-7.

58. Gandal MJ, Zhang P, Hadjimichael E, Walker RL, Chen C, Liu S, et al. Transcriptome-wide isoform-level dysregulation in ASD, schizophrenia, and bipolar disorder. Science. 2018;362:eaat8127.

59. Voineagu I, Wang X, Johnston P, Lowe JK, Tian Y, Horvath S, et al. Transcriptomic analysis of autistic brain reveals convergent molecular pathology. Nature. 2011;474:380-4. 
60. Gupta S, Ellis SE, Ashar FN, Moes A, Bader JS, Zhan J, et al. Transcriptome analysis reveals dysregulation of innate immune response genes and neuronal activity-dependent genes in autism. Nat Commun. 2014;5:5748.

61. Parikshak NN, Swarup V, Belgard TG, Irimia M, Ramaswami G, Gandal MJ, et al. Genome-wide changes in lncRNA, splicing, and regional gene expression patterns in autism. Nature. 2016; 540:423-7.

62. International Schizophrenia C. Rare chromosomal deletions and duplications increase risk of schizophrenia. Nature. 2008; 455:237-41.

63. Ng MY, Levinson DF, Faraone SV, Suarez BK, DeLisi LE, Arinami T, et al. Meta-analysis of 32 genome-wide linkage studies of schizophrenia. Mol Psychiatry. 2009;14:774-85.

64. Lewis CM, Levinson DF, Wise LH, DeLisi LE, Straub RE, Hovatta I, et al. Genome scan meta-analysis of schizophrenia and bipolar disorder, part II: schizophrenia. Am J Hum Genet. 2003;73:34-48.

65. Allen NC, Bagade S, McQueen MB, Ioannidis JP, Kavvoura FK, Khoury MJ, et al. Systematic meta-analyses and field synopsis of genetic association studies in schizophrenia: the SzGene database. Nat Genet. 2008;40:827-34.

66. Ayalew M, Le-Niculescu H, Levey DF, Jain N, Changala B, Patel $\mathrm{SD}$, et al. Convergent functional genomics of schizophrenia: from comprehensive understanding to genetic risk prediction. Mol Psychiatry. 2012;17:887-905.

67. He X, Fuller CK, Song Y, Meng Q, Zhang B, Yang X, et al. Sherlock: detecting gene-disease associations by matching patterns of expression QTL and GWAS. Am J Hum Genet. 2013;92:667-80.

68. Luo X, Huang L, Han L, Luo Z, Hu F, Tieu R, et al. Systematic prioritization and integrative analysis of copy number variations in schizophrenia reveal key schizophrenia susceptibility genes. Schizophr Bull. 2014;40:1285-99.

69. Chen C, Cheng L, Grennan K, Pibiri F, Zhang C, Badner JA, et al. Two gene co-expression modules differentiate psychotics and controls. Mol Psychiatry. 2013;18:1308-14.

70. Phipson B, Maksimovic J, Oshlack A. missMethyl: an R package for analyzing data from Illumina's HumanMethylation450 platform. Bioinformatics. 2016;32:286-8.

71. Wang J, Vasaikar S, Shi Z, Greer M, Zhang B. WebGestalt 2017: a more comprehensive, powerful, flexible and interactive gene set enrichment analysis toolkit. Nucleic Acids Res. 2017;45(W1): W130-W137.

72. Bipolar D, Schizophrenia Working Group of the Psychiatric Genomics Consortium. Electronic address drve, Bipolar D, Schizophrenia Working Group of the Psychiatric Genomics C. Genomic Dissection of Bipolar Disorder and Schizophrenia, Including 28 Subphenotypes. Cell. 2018;173:1705-15 e1716.

73. Lei H, Yan Z, Sun X, Zhang Y, Wang J, Ma C, et al. Axon guidance pathways served as common targets for human speech/language evolution and related disorders. Brain Lang. 2017;174:1-8.

74. Wefers B, Hitz C, Holter SM, Trumbach D, Hansen J, Weber P, et al. MAPK signaling determines anxiety in the juvenile mouse brain but depression-like behavior in adults. PLoS ONE. 2012;7: e35035.

75. Berridge MJ. Calcium signalling and psychiatric disease: bipolar disorder and schizophrenia. Cell Tissue Res. 2014;357:477-92.

76. Labonte B, Engmann O, Purushothaman I, Menard C, Wang J, Tan C, et al. Sex-specific transcriptional signatures in human depression. Nat Med. 2017;23:1102-11.

77. Petrovski S, Wang Q, Heinzen EL, Allen AS, Goldstein DB. Genic intolerance to functional variation and the interpretation of personal genomes. PLoS Genet. 2013;9:e1003709. 